\title{
SOME TRANSIENT RESULTS ON THE M/SM/1 SPECIAL SEMI-MARKOV MODEL IN RISK AND QUEUEING THEORIES
}

\author{
Jacques JANSSEN \\ Université Libre de Bruxelles
}

\begin{abstract}
We consider a usual situation in risk theory for which the arrival process is a Poisson process and the claim process a positive $(J-X)$ process inducing a semiMarkov process. The equivalent in queueing theory is the M/SM/ 1 model introduced for the first time by Neuts (1966).

For both models, we give an explicit expression of the probability of non-ruin on $[0, t]$ starting with $u$ as initial reserve and of the waiting time distribution of the last customer arrived before $t$. "Explicit expression" means in terms of the matrix of the aggregate claims distributions.
\end{abstract}

\section{THE SPECIAL SEMI-MARKOV MODEL IN RISK THEORY}

In a usual situation of the theory of risk, let $\left(A_{n}, n \geqslant 1\right)$ be the claim interarrival times process, $\left(B_{n}, n \geqslant 1\right)$ the claim amounts process. Moreover, we suppose that $m$ "types" of claims are possible represented by the set:

$$
I=\{1,2, \ldots, m\} \text { (with } 1 \leqslant m<\infty \text { ). }
$$

The process starts just after payment of an initial claim of type $J_{0}=i$ and after this payment, the fortune of the company is supposed to be $u(u \geqslant 0)$. The process $\left(J_{n}, n \geqslant 0\right)$ represents the sequence of the successive types of claims. For the simplicity of notations, we also introduce the random variables $A_{0}$ and $B_{0}$ such that:

$$
A_{0}=B_{0}=\text { o } \quad \text { a.s. }
$$

If the claim arrivals process is not explosive, let $N_{t}$ denote the total number of claims in $(o, t)$ (thus excluded the initial claim) and define:

$$
\left.X(t)=\sum_{n=0}^{N(t)} B_{n} \text { (total amount of claims paid on }(0, t)\right)
$$

$$
Z_{t}=J_{N(t)} \text { (type of the last claim occurred before or at } t \text { ). }
$$

If we also suppose that the incomes of the company occur at a constant rate $c(c>0)$, then the "fortune" $Z(t)$ of the company at time $t$ is given by

$$
Z(t)=u+c t-X(t) .
$$

The matrix $m x m$ of the "distribution" functions of the aggregate claims at time $t$ will be, by definition:

$$
\mathbb{f}(x, t)=\left(F_{i j}(x, t)\right)
$$


where

$$
\begin{gathered}
F_{i j}(x, t)=P\left[X(t) \leqslant x, J_{N(t)}=j \mid J_{0}=i\right] \\
(i, j=1, \ldots, m) .
\end{gathered}
$$

\section{Probabilistic assumptions}

We assume that the processes introduced satisfy the following assumptions:

1. The claim arrival process is a Poisson process of parameter $\lambda$.

2. The process $\left(\left(J_{n}, B_{n}\right), n \geqslant 0\right)$ is a positive $(J-X)$ process (see Janssen (1970)); this means that

$$
P\left[B_{n} \leqslant x, J_{n}=j \mid\left(J_{k}, B_{k}\right), k \leqslant n-1\right]=Q_{J_{n-1} j}(x) \text { a.s. }
$$

where the matrix $\mathbb{R}$, defined by $\mathbb{Q}(x)=\left(Q_{i j}(x)\right)$ is a matrix of mass functions such that:

$$
\text { i. } Q_{i j}(x)=0 \text { for all } x \leqslant \text { o for all } i, j \in I
$$

ii. $\sum_{j=1}^{m} Q_{i j}(+\infty)=1$ for all $i \in I$.

From the semi-Markov theory (PYKE (1961)), it is well-known that

$1^{\circ}$ if $p_{i j}=\lim _{x \rightarrow \infty} Q_{i j}(x)$ and $\mathbf{P}=\left(p_{i j}\right)$, then the process $\left(J_{n}, n \geqslant 0\right)$

- i.e. the process of claim types-is a homogeneous Markov chain with $\mathbf{P}$ as transition matrix.

$2^{\circ}$ The random variables $B_{n}, n \geqslant 0$ are not independent, but only conditionally dependent given the Markov chain $\left(J_{n}, n \geqslant 0\right)$

- often called the "imbedded Markov chain".

3. The processes $\left(A_{n}, n \geqslant 0\right)$ and $\left(\left(J_{n}, B_{n}\right), n \geqslant 0\right)$ are independent.

\section{The main problem}

The event "ruin before $t$ " occurs if the trajectory of $Z\left(t^{\prime}\right)$ on $(0, t)$ goes under the time axis before $t$. More precisely, if $\phi_{i j}(u, t)$ represents the probability of noll-ruin on $[0, t]$, starting with $J_{0}=i$ and an initial fortune $u$, and such that $J_{N(t)}=j$, we have, by definition:

$$
\phi_{i j}(u, t)=P\left[Z\left(t^{\prime}\right) \geqslant 0,0 \leqslant t^{\prime} \leqslant t, J_{N(t)}=j \mid J_{0}=i\right]
$$

or equivalently by (1.5):

$$
\phi_{i j}(u, t)=P\left[\sup _{0 \leqslant \tau \leqslant t}(X(\tau)-c \tau) \leqslant u, J_{N(t)}=j \mid J_{0}=i\right] .
$$


If we are not interested by the last type observed before $t$, we have enough with

$$
\phi_{i}(u, t)=\sum_{j=1}^{m} \phi_{i j}(u, t)
$$

and if $\left(p_{1}, \ldots, p_{m}\right)$ is an initial distribution on $J_{0}$, we have to compute

$$
\phi(u, t)=\sum_{j=1}^{m} p_{i} \phi_{i}(u, t) .
$$

The problem solved in this paper is to find an explicit expression of the matrix $\phi$, defined by

$$
\phi(x, t)=\left(\phi_{i j}(x, t)\right)
$$

in terms of the matrix

2. THE ANALOGOUS MODEL IN QUEUEING THEORY: THE M/SM/1 MODEL As quoted by several authors (PRABHu (1961), SEAL (1972), JANSSEN (1977)), a risk model can easily be interpreted as a queueing model and vice versa. It suffices to see the process $\left(A_{n}, n \geqslant 1\right)$ as the one of the interarrival times between two successive customers (i.e. customers $(n-1)$ and $n$ ) in a queueing system with one server and as discipline rule FIFO; then, the process $\left(B_{n}\right.$, $n \geqslant 1$ ) represents the successive service times (i.e. $B_{n}$ is the service time of the customer number $(n-1), n \geqslant 1)$.

We also suppose that at $t=0$, the customer number o just begins his service. Moreover, we have $m$ types of customers and $J_{n}$ represents the type of customer $n$. Here $N_{t}$ gives the "number" of the last customer arrived before or at $t$. With the same probabilistic assumptions as those of the preceding paragraph, the main problem considered in the queueing optic is to get an explicit expression of the distribution of $W_{N(t)}$ where $W_{n}(n \geqslant 0)$ represents the waiting of the $n$th customer. More precisely, we must express the matrix $\mathbf{W}$ in terms of where it is defined by

$$
\mathbf{W}(x, t)=\left(\mathbf{W}_{i j}(x, t)\right)
$$

with

$$
\mathrm{W}_{i j}(x, t)=P\left[\mathrm{~W}_{N(t)} \leqslant x, J_{N(t)}=j \mid J_{0}=i\right] .
$$

This model is noted $\mathrm{M} / \mathrm{SM} / 1$ in the queueing literature (Poisson arrivals and semi-Markov service times) introduced by NEUTS (1966).

\section{THE DISTRIBUTION OF AGGREGATE CLAIMS}

Introduce the usual notation in semi-Markov theory: for any matrix $m x m$ of mass functions $\mathbf{L}$, we note by $\mathbf{L}^{(\bar{n})}$ the $n$-fold convolution of the matrix $\mathbf{L}$, 
that is

$$
\mathbf{L}^{(\overline{0})}(x)=\left(U_{0}(x)\right), \mathbf{L}^{(\overline{1})}(x)=\left(L_{i j}(x)\right)
$$

(where $U_{0}(x)$ is the distribution function with a unit mass at o) and for $\mathbf{L}^{(\bar{n})}$ we have:

$$
L_{i j}^{(\bar{n})}(x)=\sum_{k \mathrm{R}} L_{i k}^{(\overline{n-1)}}(x-y) d L_{k j}(y), n \geqslant 1 .
$$

If

$$
S_{n}=\sum_{i=0}^{n} B_{i}
$$

it is clear, from (1.8), that

$$
Q_{i j}^{(\bar{n})}(x)=\mathbf{R}\left[S_{n} \leqslant x, J_{n}=j \mid J_{0}=i\right] .
$$

From assumption (3), it follows then that:

$$
\mathbb{N}^{*}(x, t)=\sum_{n=0}^{\infty} e^{-\lambda t} \frac{(\lambda t)^{n}}{n !} \mathbb{Q}^{(\bar{n})}(x)
$$

expression given the matrix of distribution of aggregate claims by means of the semi-Markov kernel $\mathbb{Q}$.

Let us remark that the assumption (1) gives:

$$
\begin{aligned}
& \mathrm{P}\left[X(t+s) \leqslant x, J_{N(t+s)}=j \mid X\left(s^{\prime}\right), J_{N\left(s^{\prime}\right)}, s^{\prime} \leqslant s, X(s)=y,\right. \\
& \left.J_{N(s)}=i\right]=F_{i j}(x-y, t)
\end{aligned}
$$

showing that the process $\left(\left(X(t), J_{N(t)}\right), t \geqslant 0\right)$ is markovian.

\section{LOADINGS OF PREMIUMS}

To show how the concept of loading of premiums can be introduced in the special semi-Markov risk model considered here, let us suppose that the quantities-mean cost of a claim of type $i-$

$$
\eta_{i}=\Sigma \int_{0}^{\infty} x d Q_{i j}(x), \quad i \in I
$$

are finite. Moreover, we suppose that the Markov chain $\left(J_{n}, n \geqslant 0\right)$ is ergodic and that $\left(\Pi_{1}, \ldots, \Pi_{m}\right)$ represents the unique stationary probability distribution. Starting with this distribution for $J_{0}$, we get, using (3.5):

$$
\begin{aligned}
\mathrm{P}[X(t) \leqslant x] & =\sum_{i} \sum_{j} \Pi_{i} F_{i j}(x, t) \\
& =\sum_{n=0}^{\infty} \sum_{i=1}^{m} \sum_{j=1}^{m} e^{-\lambda t} \frac{(\lambda t)^{n}}{n !} \Pi_{i} Q_{i j}^{(\bar{n})}(x)
\end{aligned}
$$


so that the mean of the aggregate claims at time $t$ is given by

$$
\mathrm{E}[X(t)]=\sum_{n=0}^{\infty} e^{-\lambda t} \frac{(\lambda t)^{n}}{n !}\left(\sum_{i=1}^{m} \sum_{i=1}^{n} \Pi_{i} \int_{0}^{\infty} x d Q_{i j}^{(\bar{n})}(x)\right) .
$$

The term under brackets is the expectation of $S_{n}$ or, by (3.3)

$$
\sum_{k=1}^{n} \mathrm{E}\left(B_{k}\right)
$$

As the process $(J n, n \geqslant 0)$ is stationary, we have, for all $k$

$$
\mathrm{E}\left(B_{k}\right)=\sum_{l=1}^{m} \Pi_{l} \eta_{l}
$$

This gives:

$$
\mathrm{E}[X(t)]=\sum_{n=0}^{\infty} e^{-t \lambda} \frac{(\lambda t)^{n}}{n !} n\left(\sum_{-1}^{m} \Pi_{l} \eta_{l}\right)
$$

or

$$
\mathrm{E}[X(t)]=\lambda \varphi t
$$

with

$$
\varphi=\sum_{l=1}^{m} \Pi_{l} \eta_{l}
$$

It follows that the mean fortune at time $t$ is given by:

$$
(c-\lambda \varphi) t
$$

which is positive if and only if $c=\lambda \varphi(1+\eta)$, with $\eta>0$. The justification of the loading $\eta$ comes also from the fact that, except some degenerate cases, there exists a reserve $u$ such that for all $i, j, \phi_{i j}(u)$ is positive-where $\phi_{i j}(u)$ $=\lim _{t} \phi_{i j}(u, t)$-if and only if $\lambda \varphi<c$ (see JANSSEN (1970)).

\section{EXPRESSION OF $\phi_{i j}(u, t)$}

The assumptions made-(1), (2), (3)-are such that the method used by PrabHu (1961) and later by Seal (1974) is valid. For the facility, let us suppose that the mass functions $Q_{i j}(x)$ have densities $q_{i j}(x)$ on $(0, \infty)$; then the PRABHU's integral equation becomes the integral system:

$$
\begin{array}{r}
F_{i j}(u+c t, t)=\phi_{i j}(u, t)+\sum_{k=1}^{m} \int_{0}^{i} \phi_{k j}\left(0, t-\tau d_{x} F_{i k}(u+c \tau, \tau)\right. \\
i, j=1, \ldots, m
\end{array}
$$


where

$$
d_{x} F_{i k}(u+c \tau, \tau)=c \frac{\partial F_{i k}}{\partial x}(u+c \tau, \tau) d \tau
$$

The system (5.1) gives the $\phi_{i j}(u, t)$ provided we know the values at $u=0$. These can be computed using (5.1) with $u=0$ :

$$
\begin{array}{r}
F_{i j}(c t, t)=\phi_{i j}(0, t)+\sum_{k=1}^{m} \int_{0}^{t} \phi_{k j}(0, t-\tau) d_{x} F_{i k}(c \tau, \tau) \\
i, j=1, \ldots, m .
\end{array}
$$

To write this system of Volterra integral equations in a more concise way, let us introduce the following matrices:

$$
\begin{gathered}
\phi(t)=\left(\phi_{i j}(0, t)\right)=(\phi(o, t)) \\
\mathbf{F}(t)=\left(F_{i j}(c t, t)\right)=(\mathbb{F}(c t, t)) \\
\mathbf{G}(t)=c\left(\frac{\partial F_{i j}}{\partial x}(c t, t)\right) \\
(\mathbf{A} * \mathbf{B})(t)=\left(\sum_{k=1}^{m} \int_{0}^{t} A_{i k}(t-v) B_{k j}(v) d v\right)
\end{gathered}
$$

(with $A$ and $B m x m$ matrices)

$$
\tilde{\mathbf{A}}(s)=\left(\int_{0}^{\infty} e^{-s t} A_{i j}(t) d t\right)
$$

(Laplace transform for matrices).

The system (5.3) takes the matrix form:

$$
\mathbf{F}(t)=\phi(t)+\mathbf{G} * \phi(t)
$$

and using Laplace transforms, we get

$$
\tilde{\mathbf{F}}(s)=(\tilde{\mathbf{I}}+\mathbf{G}(s)) \tilde{\phi}(s)
$$

and consequently:

$$
\tilde{\phi}(s)=(\mathbf{I}+\tilde{\mathbf{G}}(s))^{-1} \tilde{\mathbf{F}}(s)
$$

provided the inverse matrix of $\mathbf{I}+\tilde{\mathbf{G}}(s)$ exists.

We can now show the main result and for simplicity, we suppose that the derivatives $q_{i j}(x)$ of $Q_{i j}(x)$ exist for all $i$ and $j$. 


\section{Proposition}

If the quantity $M$ defined by

$$
M=\sup \left\{q_{i j}(x), i, j \in I, x \geqslant 0\right\}
$$

is finite, then

$$
\begin{gathered}
\phi(t)=\sum_{n=0}^{\infty}(-1)^{n} \mathbf{G}^{(n)} * \mathbf{F}(t) \\
\phi(u, t)=\mathbf{F}(u+c t, t)-\mathbf{G}_{u} * \sum_{n=0}^{\infty}(-1)^{n} \mathbf{G}^{(n)^{1)}} * \mathbf{F}(t)
\end{gathered}
$$

where

$$
\mathbf{G}_{u}(t)=\left(c \frac{\partial F_{i j}}{\partial x}(u+c t, t)\right) .
$$

Proof: From (3.5), we deduce that

$$
\frac{\partial F_{i j}}{\partial x}(x, t)=\sum_{n=1}^{\infty} e^{-\lambda t} \frac{(\lambda t)^{n}}{n !} q_{i j}^{(n)}(x)
$$

where

$$
q_{i j}^{(1)}(x)=q_{i j}(x)
$$

and

$$
q_{i j}^{(n)}(x)=\sum_{k} \int_{0}^{x} q_{i k}^{(n-1)}(x-y) q_{k j}(y) d y, n>1 .
$$

From (5.12), (5.18), it is clear that, for all $n \geqslant 1$

$$
q_{i j}^{(n)}(x) \leqslant M
$$

so that from (5.16):

$$
\frac{\partial F_{i j}}{\partial x}(x, t) \leqslant M\left(1-e^{-\lambda t}\right) \leqslant M .
$$

From the definition (5.6), we get

$$
\tilde{G}_{i j}(s) \leqslant c \int_{0}^{\infty} M e^{-s t} d t=\frac{c M}{s}
$$

1 From now, this symbol means the $n$-fold convolution product for the definition $(5 \cdot 7)$. 


$$
\begin{aligned}
& \tilde{G}_{i j}^{2}(s)=\Sigma \tilde{G}_{i k}(s) \tilde{G}_{k j}(s) \leqslant m \frac{c^{2} M^{2}}{s^{2}} \\
& \vdots \\
& \tilde{G}_{i j}^{n}(s)=\Sigma \tilde{G}_{i k}^{n-1}(s) \quad \tilde{G}_{k j}(s) \leqslant m^{n-1} \frac{c^{n} M^{n}}{s^{n}} .
\end{aligned}
$$

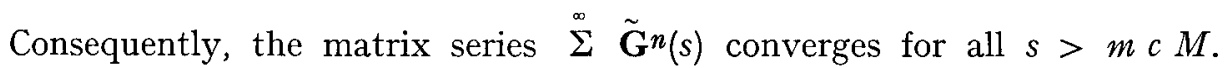
A well-known consequence of this fact is that the matrix $(\mathbf{I}+\tilde{\mathbf{G}}(s))^{-1}$ is invertible and

$$
(\mathbf{I}+\tilde{\mathbf{G}}(s))^{-1}=\sum_{n=0}^{\infty}(-1)^{n} \tilde{\mathbf{G}}^{n}(s)
$$

of course on $(m c M, \infty)$.

Using the matrix version of a theorem of DoETsch (1974) and (5.11), we get (5.13).

The result (5.14) follows then from the relations (5.1) written under the matrix form and where $\phi(t)$ is under the form (5.13).

6. RESUlTS FOR THE ACTUAL WAITING TIME AT TIME $t$ OF THE M/SM/I QUEUEING MODEL

The probabilistic assumptions made in the paragraph 1 imply that the process $\left(\left(J_{n}, A_{n}, B_{n}\right), n \geqslant 0\right)$ is a two-dimensional $(J-X)$ process (Janssen, 1979) with kernel $\left(Q_{i j}(t, x)\right)$ given by:

$$
Q_{i j}(t, x)=E(t) \cdot Q_{i j}(x)
$$

where

$$
E(t)=\left\{\begin{array}{l}
0, t<0 \\
1-e^{-\lambda t}, t \geqslant 0 .
\end{array}\right.
$$

If we suppose that the matrix $\mathbf{P}(=\mathbb{2}(+\infty))$ is ergodic with a stationary probability distribution $\left(\Pi_{1}, \ldots, \Pi_{m}\right)$, the dual kernel $\left(\hat{Q}_{i j}(t, x)\right)$ of $\left(Q_{\imath j}(t, x)\right)$ is given by (see JANSSEN (1979)):

$$
\begin{aligned}
\hat{Q}_{i j}(t, x) & =\frac{\Pi_{j}}{\Pi_{i}} Q_{i j}(t, x) \\
& =\frac{\Pi_{j}}{\Pi_{i}} E(\tau) Q_{j i}(x) .
\end{aligned}
$$


Let us now consider the $\mathrm{M} / \mathrm{SM} / 1$ queueing model whose kernel is given by (6.4). The asymptotical study has been done for the first time by NEuTs (1966). Now the transient behaviour of $\hat{W}_{i j}(x, \tau)$-defined by $(2.2)$-can be easily deduced from the last paragraph and our duality results (JANSSEN, 1979). From the proposition 4 of this last reference, we get, for all $x>0$ and all $t>0$ :

$$
\Pi_{i} \int_{0}^{1} e^{\lambda t} \hat{W}_{i j}(x, d \tau)=\Pi_{j} \int_{0}^{1} e^{\lambda t} \phi_{j i}(x, d \tau)
$$

so that

$$
\hat{W}_{i j}(x, \tau)=\frac{\Pi_{j}}{\Pi_{i}} \phi_{j i}(x, \tau)
$$

If $\Pi_{d}$ represents the $m x m$ diagonal matrix whose $i$ th element on the principal diagonal is $\Pi_{i},(6.6)$ takes the form

$$
\hat{\mathbf{W}}(x, \tau)=\Pi_{d}^{-1} \phi^{\tau}(x, \tau) \Pi_{d}
$$

with

$$
\hat{\mathbf{W}}(x, \tau)=\left(\hat{W}_{i j}(x, \tau)\right)
$$

(6.7) with the aid of (5.14) gives an explicit expression of the distribution of the actual waiting time in a $\mathrm{M} / \mathrm{SM} / 1$ model.

\section{COMMENTS}

a) For $m=1$, the model considered becomes the classical Cramér's model of risk theory and the $M / G / 1$ queueing model for which it is known (see PRABHU (1961), SEAL (1972)) that:

$$
\phi(0, t)=\frac{1}{t} \int_{0}^{t} F(x, t) d x .
$$

Using successive integrations by parts, it is possible to show-in this casethe equivalence of (7.1) and (5.13). It does not seem possible to have an analogous result for $m>1$, in particular an extension of the analytically proof of DE VyLDER (1977) cannot be used as the variables $\left(B_{n}\right)$ are no more exchangeable.

b) The effect of a suppression of the $k^{e}$ type of claim is theoretically possible by comparing $\phi(u, t)$ and $\phi_{k}(u, t)$, representing the non-ruin probability with $(m-1)$ types of claims, $k$ being excluded. 
c) The main result can be extended to the non-Poisson case if we suppose that the process $\left(J_{n}, A_{n}\right)$ is a semi-Markov process of kernel

$$
\left(p_{i j} E_{i}(t)\right)
$$

where

$$
E_{i}(t)= \begin{cases}0 & , t<0 \\ 1-e^{-\lambda_{i} t}, t \geqslant 0\end{cases}
$$

that is a regular continuous Markov process with a finite number of states.

d) The following remarks may be useful for numerical computation.

It is easy to show that

$$
\mathbf{G}^{(n)} * \mathbf{F}(t) \leqslant m^{n} \frac{M^{n} t^{n}}{n !}
$$

so that approximating $\phi(t)$ by the first $(N-1)$ terms of $(5.13)$, we have for the absolue value of the error $R_{N}(t)$, the following upper bound:

$$
\left|R_{N}(t)\right| \leqslant \frac{(m M t)^{N}}{N !} e^{m M t}
$$

For $m=1$, we can say more. Indeed, let us suppose, without loss of generality, that $c=1$ and $M \leqslant 1$. For $c$, that is well-known in risk theory; if $M>1$, it suffices to introduce the random variables $\left(B_{n}^{\prime}\right),\left(A_{n}^{\prime}\right)$ defined by $B_{n}^{\prime}=M^{-1} B_{n}$ and $A_{n}^{\prime}=M^{-1} A_{n}$ so that the process $\left(A_{n}^{\prime}\right)$ induces a Poisson one of parameter $\lambda^{\prime}=M^{-1} \lambda$. Then, if $\phi^{\prime}\left(u^{\prime}, t^{\prime}\right)$ is the probability of non-ruin for this model: $\phi(u, t)=\phi^{\prime}(M u, M t)$. (7.4)

In this case, we have

$$
\mathbf{G}^{(n)} * \mathbf{F}(t)-\mathbf{G}^{(n+1)} * \mathbf{F}(t)=\mathbf{G}^{(n)} *\left(U_{0}-\mathbf{G}\right) * \mathbf{F}(t)
$$

which is a non-negative quantity as $G(t) \leqslant 1$ ( $U_{0}$ is the Heaviside function with a unit mass at o).

Consequently, the series $(5.13)$ is alternating so that the sign of the error $R_{N}$ is this of $(-1)^{N}$ and

$$
\left|R_{N}(t)\right| \leqslant \mathbf{G}^{(N)} * \mathbf{F}(t) .
$$

From (7.2), it follows that:

$$
\left|R_{N}(t)\right| \leqslant \frac{t^{N}}{N !}
$$


REFERENCES

De Vylder, F. (1977). A new proof for a known result in risk theory. J. of Comp. Ap. Math., 3, 277-279.

Doetsch, G. (1974). Introduction to the theory and application of the Laplace transform. Springer-Verlag, Berlin.

JANSSEN, J. (1970). Sur une généralisation du concept de promenade aléatoire sur la droite réelle. Ann. Inst. H. Poincaré, B, VI, 249-269.

Janssen, J. (1977). The semi-Markov model in risk theory, in Advances in Operations Research edited by M. Roubens, North-Holland, Amsterdam.

Janssen, J. (1979). Some explicit results for semi-Markov in risk theory and in queueing theory. Operations Research Verfahren 33, 217-231.

Pyke, R. (1961). Markov Renewal Processes: Definitions and preliminary properties. Ann. Math. Statist. 32, 1231-1242.

Prabhu, N. U. (1961). On the ruin problem of collective risk theory. Ann. Math. Statist. 32, $757-764$.

Neuts, M. F. (1966). The single server queue with Poisson input and semi-Markov service times. J. Appl. Prob. 3, 202-230.

Seal, H. L. (1972). Risk theory and the single server queue. Mitt. Verein. Schweiz. Versich. Math. 72, 171-178.

SeAL, H. L. (1974). The numerical calculation of $U(W, t)$, the probability of non-ruin in an interval (o, t). Scand. Actu. J. 1974, 121-139. 